\section{Histological \\ measurement of retinal nerve fibre layer thickness}

\begin{abstract}
Purpose Accurate assessment of the retinal nerve fibre layer (RNFL) is central to the diagnosis and follow-up of glaucoma. The in vivo measurement of RNFL thickness by a variety of digital imaging technologies is becoming an important measure for early detection, as well as for follow-up, of glaucomatous damage. However, when drawing clinical inference concerning the state of the RNFL, it is important to have valid reference data on RNFL thickness in both healthy and diseased eyes. In this review, we summarize the knowledge currently available about RNFL thickness in human and primate eyes.
\end{abstract}

Methods A review of the literature on histological analysis of RNFL thickness in the context of glaucomatous damage. Conclusions Six studies have so far analysed RNFL thickness. Despite the diverse study methodology taken, a consistent feature of all the data is that the superior and inferior quadrants of the peripapillary retina are thicker than the nasal and temporal quadrants; that the RNFL thickness rapidly diminishes with increasing distance from the disc margin; and that apparently at different locations the ratio of axons to supportive tissue varies significantly. We conclude that limited data are available to describe the normal variation in RNFL thickness in the normal human eye. Further studies may help better characterize the RNFL thickness in health and disease and to facilitate the correlation with clinical methods for nerve fibre layer assessment.

Eye (2005) 19, 491-498. doi:10.1038/sj.eye.6701569

Published online 27 August 2004

Keywords: retinal nerve fibre layer; retina; glaucoma; histology; optic nerve; ganglion cell

\section{Introduction}

The last decade has seen a dramatic increase in the number of articles addressing the clinical estimation of retinal nerve fibre layer (RNFL) thickness in glaucoma. The measurement of this parameter may play a central role in the early detection of glaucomatous damage given the changes that can be seen in the RNFL prior to the onset of visual field loss. ${ }^{1-5}$ In parallel, the implementation of RNFL evaluation both by clinical assessment as well as with imaging devices in routine clinical practice of glaucoma patients is on the rise. By contrast, we know relatively little about histological estimates of RNFL thickness in normal and diseased human eyes.

While each of the commercially available imaging devices (confocal scanning laser ophthalmoscopy, scanning laser polarimetry, optical coherence tomography ${ }^{2}$ ) has had its own set of normative RNFL thickness values collected, these values have not been validated histologically. While clinical studies have shown that these devices hold considerable clinical promise in the detection of disease, the extent to which they provide a true estimate of RNFL thickness is not certain. The paucity of histological data may reflect a view that accurate measurement of the RNFL is secondary to the ability of any given device to diagnose disease. In clinical terms, this approach may have some merit. However, the lack of clinical-histological correlations limits comparison between devices and can hinder the recognition of imaging artefacts that might arise with some RNFL measurement techniques. Given the potential importance of RNFL thickness, the paucity of high-quality data on retinal nerve fibre thickness is a drawback. In part it reflects technical difficulties in the histological analysis of the RNFL. In this review, some of these issues are highlighted and discussed.
${ }^{1}$ Department of Ophthalmology, Hebrew University - Hadassah Medical Center, Jerusalem Israel

${ }^{2}$ University of Wales College of Medicine, Heath Park Cardiff, UK

Correspondence: EZ Blumenthal Department of Ophthalmology, Hadassah University Hospital PO Box 12000 Jerusalem 91120, Israel Tel: + 972-2-677-6580 Fax: + 972-2-675-8926 E-mail: eblumenthal@ md.huji.ac.il

Received: 16 January 2004 Accepted: 12 March 2004 Published online: 27 August 2004 


\section{Approaches for quantifying glaucomatous damage histologically}

Before focusing on the RNFL thickness as a means for quantifying glaucomatous damage histologically, we would like to acknowledge that different approaches exist for quantifying ganglion cells and their axons histologically in the enucleated eye.

\section{Ganglion cell body counts}

Death of ganglion cells is the common final pathway event in glaucoma pathophysiology. Ganglion cell dropout can therefore provide an accurate assessment of the damage inflicted by the disease process. Two different approaches utilizing ganglion cell counts, come to mind: first, total counts or density maps of ganglion cell bodies. Second, staining diseased or dying cells in the ganglion cell layer, such as apoptotic cells stained using the TUNEL method. ${ }^{6,7}$ Visualizing dying ganglion cells can provide powerful insight to disease presence and rate of loss at the moment of tissue harvesting. While an appealing approach, total retinal ganglion cell counts have been performed on very few human eyes, most of them normal. ${ }^{8-12}$ This histological approach, while potentially powerful, is extremely labour intensive.

\section{Optic nerve axonal counts}

This is a common histological approach that has been used as an endpoint for evaluating glaucomatous damage in animal models, ${ }^{13}$ as well as in healthy ${ }^{14-16}$ and glaucomatous ${ }^{17}$ human eyes. Axons in the optic nerve can be relatively easily stained and visualized owing to the myelin sheath surrounding each individual axon; such a sheath is lacking from the intraocular (RNFL) portion of the ganglion cell axon. Gross topographic representation of the retina in the optic nerve cross-section might enable, though to a very limited extent, identification of focal damage. ${ }^{18}$ This technique is labour intensive even when computerized imaging analysis tools have automated portions of the task. There appears to be an approximately two-fold interindividual variability in the total nerve fibre counts of normal optic nerves, ${ }_{1}^{19}$ somewhat limiting the ability of this approach to detect damage in the optic nerve when analysed at a single point in time. In contrast, Balazsi et al. ${ }^{14}$ found far less variability in total axonal counts of normal human optic nerves.

\section{Optic nerve head morphometry - a histological evaluation}

Clinically, photographic and imaging analyses of the optic nerve head topography are standard approaches in glaucoma diagnosis and follow-up, far more prevalent than analysis of the RNFL. ${ }^{2,20}$ A few studies have reported the approximate cup-to-disc ratio of the optic disc head in enucleated eyes as a means of differentiating normal from glaucomatous eyes. ${ }^{13,21}$ Nevertheless, we are not aware of a study providing an in-depth evaluation of the morphometry of the optic disc head in histological sections of enucleated eyes. This is perhaps owing to the topographical changes, most notably swelling, seen at the level of the optic disc, occurring shortly after death.

\section{RNFL thickness}

As compared to optic nerve axonal counts that can accurately sum up the total number of ganglion cells in the eye, but without providing information on focal dropout, RNFL thickness can provide data on focal RNFL loss, and hence on focal ganglion cell loss. This can be achieved by peripapillary measurements of the RNFL thickness, owing to the retinotopic organization of the nerve bundles entering the optic disc. ${ }^{22,23}$ The availability of RNFL thickness measurements obtained via several imaging devices in living human eyes, and the rationale in quantifying RNFL dropout via thickness measurements, makes this histological approach relevant to clinical diagnosis and follow-up, perhaps more relevant than the other three approaches discussed above.

\section{RNFL axonal counts}

A fifth approach for quantifying glaucomatous damage involves actual counts of RNFL axons. Focal axonal counts may hold promise in becoming, perhaps, a gold standard for evaluating treatment modalities in animal models. Axonal counts circumvent the assumption of a correlation between RNFL thickness and actual axonal contents, thus eliminating the contribution of the nonaxonal components of the RNFL. Axonal counts also bypass the confounder effect of calibre differences found between axons, and the possibility of a preferential loss of certain calibre axons. Lastly, axonal counts are not as affected by post-mortem and fixation (shrinkage or expansion) artefacts.

The intraocular portion of the ganglion cells' axon is not covered by myelin, therefore the calibre of some of them spans less than $1 \mu \mathrm{m}$ in diameter, bordering the resolution limits of light microscopy. Thus, identifying and counting individual axons requires the use of electron microscopy. ${ }^{24}$ It seems that the high expense and labour involved in electron microscopy as well as the need to summate enormous quantities of topographically 
aligned data are some of the reasons why this approach has not taken the lead despite its potential advantages.

\section{RNFL thickness measurements: methodological issues}

\section{Tissue considerations}

Accurate assessment and quantification of retinal structure is difficult. The principal difficulty is the period of time elapsing from death to fixation during which the retinal nerve fibre layer swells. In contrast with human tissue, such changes are not seen in animal models when the eye is perfused and fixed at the precise time of death, resulting in a clear and unswollen nerve fibre layer.

\section{Tissue selection}

Most of the data presented to date on RNFL thickness measurements comes from studies on normal eyes, both primate (several species), ${ }^{24}$ and human. ${ }^{25,26}$ Few groups report on studies comparing normal to damaged retinas, either glaucomatous damage or focal inflicted damage caused by optic nerve manipulation. ${ }^{27,28}$ The 'normal' status of some of these human globes might be questioned since they were enucleated mostly because of intraocular tumours, although these tumours are reported neither to have occluded the anterior chamber angle nor to have directly affected the inner retina or optic nerve. ${ }^{27}$ We were unable to identify any studies on RNFL thickness that have analysed seeing human eyes known to be glaucomatous. One study included 21 enucleated blind eyes with absolute secondary angleclosure glaucoma, and has provided valuable data on the residual RNFL thickness remaining once all axons have presumably disappeared. $^{27}$

\section{Time after death}

Tissue used in the articles reviewed was obtained from both primate and human sources. Primate tissue was harvested immediately after the animals were killed by the administration of a lethal dose of anaesthetic, or by perfusion fixation. Human tissue was obtained during surgical enucleation due to a malignant tumour, or from autopsies of deceased donors. Most donor eyes were harvested and fixed 0-6h post mortem. ${ }^{25,27}$ Data concerning the time interval between death and tissue fixation for some of the human specimens were not disclosed. ${ }^{25,27}$ Balazsi et al. ${ }^{14}$ addressed the influence of time from death to fixation and found it to have a significant effect on measurements of the human optic nerve. While postmortem changes might not have affected axon counts (unless autolysis occurred), they probably result in at least some shrinkage or conversely tissue swelling.

\section{Tissue preparation considerations}

Nearly every research group used its own method for fixing the specimens, including: choice of fixative chemical, embedding material (paraffin, epoxy resin, historesin, plastic), duration of fixation (48-96 h), method of sectioning (glass knife, microtome, frozen sections), thickness of sections $(1,2,60 \mu \mathrm{m})$, and method of viewing the slides (light, differential interference contrast, or electron microscopy). These differences probably led to significant differences in the amount of tissue shrinkage occurring during sample preparation. ${ }^{14}$ Ogden $^{26}$ addressed the issue of tissue shrinkage in his method of fixation and estimated it to be about $10-15 \%$.

\section{Sectioning the eye}

\section{Sectioning approach}

Each study group selected a different approach towards sectioning the globes (Figure 1). These approaches differ from one another in several ways: the retinal location selected for RNFL measurements (at the disc border, peripapillary, juxta-macular, other locations, and whole retina); the choice of knife used for sectioning; the direction of the cuts (horizontal, vertical, or other); the choice of single vs serial sectioning; the intervals between
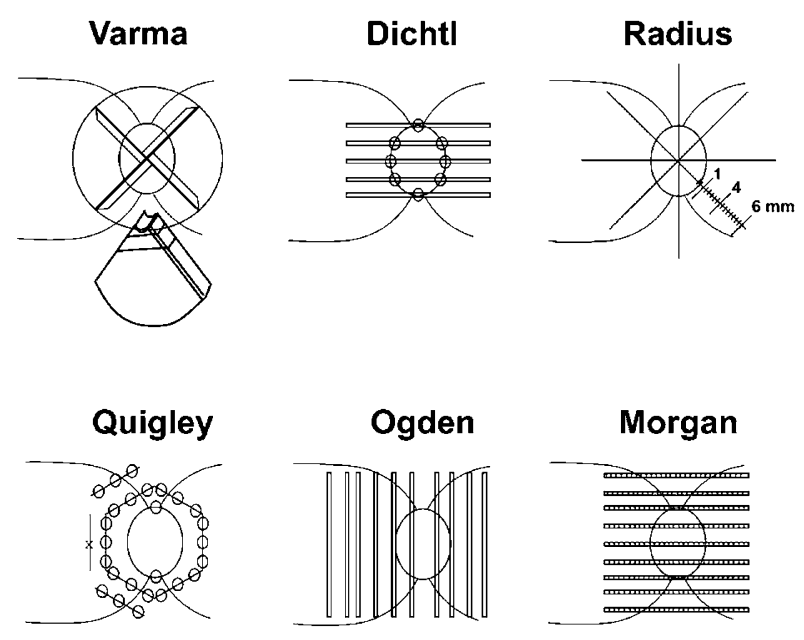

Figure 1 Schematic illustrations of the sectioning approaches used in each of the six studies: Varma trephined a $7 \mathrm{~mm}$ circle centred on the optic disc, sectioned the circle into four quadrants and then sectioned each quadrant at $50 \mu \mathrm{m}$ intervals. Dichtl sectioned the posterior pole horizontally, measuring RNFL thickness at the disc border in each section. Radius cut out rectangular retinal pieces, which he serially sectioned. Data were presented at distances of 1,4 , and $6 \mathrm{~mm}$ from the disc margin, along 8 meridians. Quigley sampled the superior and inferior disc borders, as well as along the hexagonal cuts shown. Ogden serially sectioned the disc and peripapillary retina vertically. Morgan serially sectioned the disc and peripapillary retina horizontally, measuring RNFL thickness along each section. 
sections; section thickness; and the number of measurement data points collected from each eye, as reflected by both between and within histological sections. The lack of standardization in sectioning approach (Figure 1), in addition to many other differences between the studies, such as species, makes a meaningful comparison of thickness measurements between the studies difficult. Table 1 summarizes some of these methodological differences.

\section{Section thickness}

Different sectioning approaches and type of microscope used for observation led to differences in the choice of section thickness. It is hard to determine which of the light microscopy section thickness is most appropriate: $1,2,3,6$, or even $120 \mu \mathrm{m}$. It is even more difficult to compare the above light microscopic sections to the $60 \mu \mathrm{m}$ frozen sections prepared for differential interference contrast microscopy. ${ }^{29}$ The relation of section thickness to reproducibility and accuracy of RNFL data was not addressed in any of the manuscripts.

\section{Staining considerations}

Three different staining techniques were used: PAS, toluidine blue, and paraphenylendiamine, each having unique staining properties. All staining methods are able to differentiate the RNFL from neighbouring layers accurately. However, tissue dehydration prior to staining harbours risks for tissue shrinkage, distortion, and other artefacts since the tissue has to be dehydrated and cleared before a stain can be applied. These artefacts were best avoided with the use of differential interference contrast optics, ${ }^{29}$ since tissue preparation for both light and electron microscopy requires dehydration. ${ }^{26}$

\section{Histological reconstruction}

A large number of measurements from multiple slides is required to recreate a detailed histological topographic map of the RNFL. However, when comparing RNFL imaging to histology, even a limited number of measurements provides a reasonably representative picture. ${ }^{29}$ Moreover, as mentioned above, measuring the peripapillary area should be sufficient to describe RNFL focal (wedge-shaped) changes elsewhere in the retina. Thus, once a clear map of the peripapillary zone is obtained, mapping the RNFL thickness across the entire posterior pole might not be necessary.

Most of the studies, often collecting a significant number of data points, have consequently reduced and presented the data in the format of eight peripapillary RNFL thickness measurements (Figure 2). This data reduction approach warrants caution in interpreting these maps, when compared with Ogden's more comprehensive whole retinal map, due to averaging and consequent loss of focal information, which might be 'averaged' within the four quadrants. ${ }^{26,27}$

\section{Potential artefacts}

Several factors may lead to artefacts altering the RNFL thickness measurements. These artefacts may result from tissue changes that occur after death, as well as from fixation, sectioning, and staining.

Tissue swelling

Immediately after death, owing to a loss of metabolic function of cells, the process of tissue swelling leads to an accumulation of fluid in the tissues, distorting morphology. One need only consider the changes seen in a living eye shortly after a central retinal artery occlusion, or an anterior ischaemic optic neuropathy, to comprehend the changes that might occur when the entire organism dies. Balazsi et al. ${ }^{14}$ reported increasing tissue oedema with increasing time to fixation in their optic nerve specimens when comparing $0,6,8,14,26$, and $37 \mathrm{~h}$ from death to fixation.

\section{Tissue autolysis}

From the moment of death the process of tissue autolysis begins. Autolysis is the 'self digestion' of tissue, owing to liberation and activation of various proteolytic enzymes released from intracellular compartments. This process eventually leads to loss of tissue, but can initially distort the tissue morphology as it does not necessarily affect different tissues uniformly. The effect of tissue autolysis was markedly reduced in primates when anaesthetized animals were killed by using retrograde perfusion of a fixative through the abdominal aorta. ${ }^{28}$ This procedure ensures that the tissue is fixed at the exact moment of death, before autolysis begins. In contrast, human donor tissue is usually fixed several hours postmortem leading to an unknown amount of autolytic damage to the RNFL.

\section{Tissue shrinkage}

Immediately after removal, tissues begin to shrink upon exposure to room air (reduced temperature) and continues to shrink during tissue preparation (due to dehydration). Abramson et al. . $^{30}$ quantified the shrinkage of optic nerve specimens to be in the order of $30 \%$, when comparing the situation immediately after excision to the final pathological specimen.

The staining process requires tissue dehydration. Tissue processed for both light and electron microscopy is dehydrated by passing it through graded alcohols. When water, the major constituent of cells, is removed in the dehydration process, the tissue may shrink. Further, the fixation process itself may cause additional tissue 
Table 1 Methodological differences between histological studies of RNFL thickness

\begin{tabular}{|c|c|c|c|c|c|c|c|c|c|}
\hline Author & Species & $\begin{array}{l}\text { No. of eyes } \\
\text { examined }\end{array}$ & $\begin{array}{l}\text { Type of } \\
\text { microscopy }\end{array}$ & $\begin{array}{l}\text { Thickness of } \\
\text { sections }\end{array}$ & Type of sections & No. of sections & No. of locations & Staining & Notes \\
\hline Varma (1996) & Human & 10 & Light & $3 \mu \mathrm{m}$ & Serial sections & 25 serial sections & $\begin{array}{l}\text { Eight locations at } \\
\text { three concentric } \\
\text { circles }\end{array}$ & $1 \%$ toluidine blue & \\
\hline Dichtl (1999) & Human & 3 & Light & $6 \mu \mathrm{m}$ & $\begin{array}{l}\text { Serial sections, } \\
\text { every 10th } \\
\text { examined }\end{array}$ & $\begin{array}{c}\text { 17-23 sections per } \\
\text { eye }\end{array}$ & $\begin{array}{c}\text { Eight locations at } \\
\text { the optic disc } \\
\text { margin }\end{array}$ & PAS $^{\mathrm{a}}$ & $\begin{array}{l}+19 \text { single sections } \\
\text { of different eyes } \\
+21 \text { blind } \\
\text { glaucomatous eyes }\end{array}$ \\
\hline Radius (1980) & $\begin{array}{c}\text { Owl } \\
\text { Rhesus } \\
\text { Squirrel }\end{array}$ & $\begin{array}{l}7 \\
5 \\
3\end{array}$ & Light & $2 \mu \mathrm{m}$ & $\begin{array}{l}\text { Star-shaped } \\
\text { sections, } \\
\text { serially cut }\end{array}$ & $\begin{array}{l}1,4, \text { and } 6 \mathrm{~mm} \text { from } \\
\text { disc margin }\end{array}$ & $\begin{array}{l}\text { Eight relevant } \\
\text { locations }\end{array}$ & $\begin{array}{l}\text { Paraphenylen- } \\
\text { diamine }\end{array}$ & \\
\hline Quigley (1982) & Cynomolgus & 9 & Light & & $\begin{array}{l}\text { Surrounding the } \\
\text { disc and hexagonal } \\
\text { sections }\end{array}$ & $\begin{array}{c}\text { Disc border, } 1 \mathrm{~mm} \\
\text { from border, and } \\
\text { distant }\end{array}$ & 29 locations & $\begin{array}{l}\text { Paraphenylen- } \\
\text { diamine }\end{array}$ & \\
\hline Ogden (1983) & $\begin{array}{l}\text { Cynomolgus } \\
\text { (Cynomolgus } \\
\text { + Rhesus) } \\
\text { Cynomolgus } \\
\text { + Rhesus }\end{array}$ & $\begin{array}{c}1 \text { whole map } \\
(5+2) \\
1+1\end{array}$ & $\begin{array}{l}\text { Light } \\
\text { Electron- } \\
\text { microscopy }\end{array}$ & $\begin{array}{c}120 \mu \mathrm{msections} . \\
\text { Some } \\
\text { resectioned at } \\
1 \mu \mathrm{m} \text { (glass } \\
\text { knife) } \\
1-2 \mu \mathrm{m}\end{array}$ & $\begin{array}{l}\text { Serial sections of } \\
\text { entire retina } \\
\text { Serial sections every } \\
100 \mu \mathrm{m} \text { along } \\
\text { selected bundles }\end{array}$ & & & $\begin{array}{l}\text { Toluidine blue } \\
\text { No staining } \\
\text { required }\end{array}$ & $\begin{array}{c}(5+2) \text { eyes sampled } \\
\text { at } 12-15 \text { sites, } \\
\text { compared with } \\
\text { whole mapped eye }\end{array}$ \\
\hline Morgan (1998) & Rhesus & 1 & $\begin{array}{c}\mathrm{DIC}^{\mathrm{b}} \\
\text { microscopy }\end{array}$ & $\begin{array}{l}60 \mu \mathrm{m} \text { Frozen } \\
\text { sections }\end{array}$ & $\begin{array}{c}\text { Serial sections, } \\
\text { every } 6 \text { th examined }\end{array}$ & & 216 locations & $\begin{array}{l}\text { No staining } \\
\text { required }\end{array}$ & \\
\hline
\end{tabular}

${ }^{\text {aPAS }}=$ periodic acid-Schiff method.

${ }^{\mathrm{b}} \mathrm{DIC}=$ Differential interference contrast - requires neither drying nor staining. 

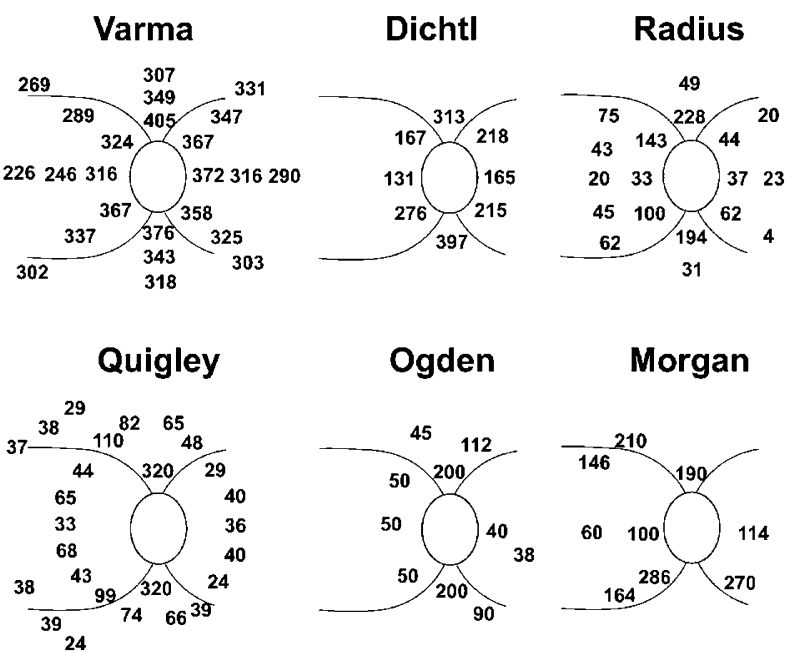

Figure 2 RNFL thickness measurements, (in $\mu \mathrm{m}$ ) found in each study, presented in right eye configuration: Varma's concentric measurement circles are $50 \mu \mathrm{m}$ apart. Dichtl's measurements are at the disc border. Radius' measurements are at 1 and $4 \mathrm{~mm}$ from the disc margin. Quigley's hexagon is $1 \mathrm{~mm}$ from the disc margin. The schemes of Ogden and Morgan give several data points at representative locations near the disc.

shrinkage, as the tissue loses its flexibility and partly in-folds upon itself. Morgan et al attempted to bypass the potential artefacts introduced during fixation and staining both by using a retrograde perfusion of fixative (often referred to as perfusion fixation rather than fixation by immersion, as discussed above), and rather than using standard fixation and staining, by using the frozen section technique. This technique assures that the cellular structures remain relatively intact and shrinkage is minimized. ${ }^{29}$

\section{Inadvertent oblique sectioning}

To arrive at valid RNFL thickness measurements, sectioning must be perpendicular to the retinal surface, otherwise the RNFL thickness measurement is artefactually increased. Furthermore, oblique sectioning of the block may also result in inaccurate topographic representation of the section.

\section{Caveats in histological RNFL thickness measurements}

Losing orientation (correct topographical representation) Near the disc margin the RNFL tapers abruptly, so that small topographical location errors translate into large thickness errors. Figure 3 in Varma et al' $\mathrm{s}^{25}$ article demonstrates that a topographic location (orientation) error as small as one RNFL thickness $(<300 \mu \mathrm{m})$ can result in approximately halving the measured RNFL thickness. Without clear landmarks in each section, it is often difficult to verify the location accurately. Through a meticulous approach, Ogden ${ }^{26}$ serially sectioned the whole retina in the vertical orientation and achieved location identification accuracy of $100 \mu \mathrm{m}$ in the horizontal plane and $25 \mu \mathrm{m}$ in the vertical plane.

Defining the RNFL borders (edge detection)

Both edges of the RNFL need to be accurately delineated as a prerequisite to meaningful RNFL thickness measurements. Large blood vessels that enter the optic disc, mainly superiorly and inferiorly, require special consideration. All authors have chosen not to measure the RNFL thickness near such vessels. A second consideration is the ability to define the outer border of the RNFL layer in areas of scattered ganglion cells that appear embedded within the RNFL. Quigley and Addicks ${ }^{28}$ defined the outer interface of the RNFL as the first ganglion cell body they came across, an approach that might lead to some underestimation of the RNFL thickness.

\section{RNFL thickness}

Six studies have, to date, quantified the thickness of the RNFL histologically in primate and human eyes. . $4,25,27-29,31^{2}$ While each used a different approach, the cumulative data define several key characteristics of the RNFL in the primate and human eye. Table 1 summarizes key aspects of each study, regarding sectioning, staining, and the type of microscopy, as discussed above. As mentioned, difficulties in comparing the numerical data presented in these studies result from the different species studied, the sectioning techniques and the locations chosen for sampling. A summary of the numerical data found is presented in Figure 2. These combined data demonstrate that the superior and inferior quadrants of the peripapillary retina are thicker than the nasal and temporal quadrants, as is expected from the site of entry of the superotemporal and inferotemporal arcuate bundles as they approach the optic disc. However, the ratio between the superior and inferior quadrants, as opposed to the nasal and temporal quadrants, differs between studies from almost $1: 1$ in the study of Varma et al. ${ }^{25}$ to $1: 4$ in Ogden's $\mathrm{s}^{26}$ whole retinal map, and up to $1: 10$ in Radius ${ }^{\prime 31}$ rhesus monkey eyes. Thickness measurements for locations away from the optic disc margin show that the RNFL thickness rapidly diminishes with increasing distance from the disc. The RNFL assumes a rather uniform 20-40 $\mu$ m layer far away from the disc. In general, RNFL thickness measurements in human retinas (noted in studies by Varma $e t a^{25}$ and Dichtl $e t a l^{27}$ ), were found to be greater than measurements obtained from primate eyes, especially so for the nasal and temporal quadrants, ${ }^{26,28,31}$ as demonstrated in Figure 2. 
Quigley and Addicks created a variety of lesions to the optic nerve of primate eyes, and examined the outcome at the RNFL level up to 3 months later. In areas where complete damage occurred, leaving a mixture of capillaries, astrocytes and Muller cells (no axons remaining), they measured the RNFL to be $10 \mu \mathrm{m}$ thick. However, the residual thickness at the disc border was $115 \mu \mathrm{m}$, compared to normal RNFL measurements of $320 \mu \mathrm{m}$ in these locations. These confusing data might imply that at different locations the ratio of axons and supportive tissue might vary significantly, at least in cynomolgus monkeys. This finding needs to be further investigated to better interpret the readouts of RNFL imaging devices; with a consideration to edge detection algorithms. $^{32}$

\section{Discussion}

This review highlights the rather limited data currently available on the anatomical features of the human RNFL. This contrasts the large body of knowledge regarding accuracy, reproducibility, and clinical efficacy of devices aimed at measuring the RNFL thickness in vivo.

Two points deserve emphasis. First, the variation in RNFL thickness around the optic nerve head is likely to differ in the human compared with primates such as the macaque (Figure 2). This observation is important since non-human primates are often used as a surrogate for studying human disease. Second, studies of RNFL thickness have been used to inform debates concerning the accuracy of devices used to measure RNFL in the human. Caution should be exercised in comparing between species, a point that has been highlighted by Ogden $^{26}$ in consideration of different patterns of RNFL organization in different primate species.

Only a handful of studies have so far measured the RNFL thickness histologically in either primate or human eyes. The cumulative data they provide enable us to construct a crude topographical map of RNFL thickness. The single most informative study is Ogden's ${ }^{26}$ whole retinal analysis performed on one cynomolgus monkey eye. Several additional studies have later confirmed his measurements and suggested a 'double-hump' configuration of the peripapillary retina. In contrast, Varma et $a l^{25}$ did not find this pattern in his data, though it is possible that the sectioning technique used might have contributed to the diminishing of this pattern. Morgan et al. ${ }^{29}$ correlated actual histology and imaging data collected on a single Rhesus (Macaca mulatto) monkey eye and showed reasonable but variable correlation between histology and imaging.

Having analysed the available literature on the topic, we conclude that the anatomical characteristics of RNFL thickness require further clarification. The studies discussed differ significantly from one another in several key aspects. Hence, it is difficult to compare across studies and species as to the numerical thickness data provided. Different species of monkeys appear to have different RNFL thickness values. ${ }^{31}$ By the same token, measurements performed on primate eyes may not be quantitatively relevant to the human RNFL. Moreover, the two studies conducted on human eyes show disagreement as to the thickness values found..$^{25,27}$

Another point for consideration is the residual thickness of the RNFL remaining after all axons have been lost. Quigley and Addicks ${ }^{28}$ have found this value to be highly variable, from $10 \mu \mathrm{m}$ in the retinal periphery, to $115 \mu \mathrm{m}$ at the disc border. This varying residual may affect the interpretation of RNFL thickness measurements obtained with imaging technology in clinical routine. In the quest for data, it is important to point out that sound histological measurements free of meaningful artefacts are unlikely to be obtainable for the reasons listed in this manuscript. Furthermore, lacking a gold standard, it may be difficult, if not impossible, to even estimate the amount of error introduced by each of the potential artefacts related to postmortem tissue changes secondary to obtaining, fixing, sectioning, and staining human tissue.

It is thus debatable as to how crucial it is to arrive at the true RNFL thickness map. Regardless, one can construct a normative database for discriminating glaucomatous eyes from normal eyes using imaging devices, based on relative, rather than absolute data. We feel that true anatomical measurements would be helpful in correlating data gathered using different imaging techniques. This knowledge may ultimately help refine clinical decisions and facilitate discrimination between different stages of glaucoma, and between normal eyes and eyes with early stages of disease. However, the numerous artefacts encountered in histological processing of the retina, towards quantifying RNFL thickness may hinder our efforts to proceed beyond a relative thickness map. A critical review of the current literature does not leave us optimistic on this issue, namely, the ability to arrive, via histology, at true RNFL thickness measurements that accurately represent the situation in the living human eye.

In conclusion, the current knowledge regarding RNFL thickness is insufficient to validate the various imaging technologies aimed at quantifying RNFL thickness in the living human eye. Further studies are required to enhance our knowledge and understanding of the normal RNFL. Once the normal topographic map of RNFL thickness is described, further issues worth tackling include the effects of aging and, perhaps of far greater significance, quantifying patterns of RNFL loss in human glaucoma. 


\section{References}

1 Zangwill LM, Williams JM, Weinreb RN. Lasers in Ophthalmology, Vol 11. Ophthalmology Clinics of North America, WB Saunders, Philadelphia, 1998, pp 233-241.

2 Blumenthal EZ, Weinreb RN. Assessment of the retinal nerve fibre layer in clinical trials of glaucoma neuroprotection. Surv Ophthalmol 2001; 45 (Suppl 3): S305-S312, discussion S332-S334.

3 Gramer E, Tausch M. Measurement of the retinal nerve fibre layer thickness in clinical routine. Curr Opin Ophthalmol 1998; 9: 77-87.

4 Kamal DS, Bunce C, Hitchings RA. Use of the GDx to detect differences in retinal nerve fibre layer thickness between normal, ocular hypertensive and early glaucomatous eyes. Eye 2000; 14 (Part 3A): 367-370.

5 Bowd C, Zangwill LM, Blumenthal EZ, Vasile C, Boehm AG, Gokhale PA et al. Imaging of the optic disc and retinal nerve fibre layer: the effects of age, optic disc area, refractive error, and gender. J Opt Soc Am A 2002; 19: 197-207.

6 Quigley HA, Nickells RW, Kerrigan LA, Pease ME, Thibault DJ, Zack DJ. Retinal ganglion cell death in experimental glaucoma and after axotomy occurs by apoptosis. Invest Ophthalmol Vis Sci 1995; 36: 774-786.

7 Kerrigan LA, Zack DJ, Quigley HA, Smith SD, Pease ME. TUNEL-positive ganglion cells in human primary openangle glaucoma. Arch Ophthalmol 1997; 115: 1031-1035.

8 Quigley HA, Dunkelberger GR, Green WR. Retinal ganglion cell atrophy correlated with automated perimetry in human eyes with glaucoma. Am J Ophthalmol 1989; 107: 453-464.

9 Curcio CA, Allen KA. Topography of ganglion cells in human retina. J Comp Neurol 1990; 300: 5-25.

10 Medeiros NE, Curcio CA. Preservation of ganglion cell layer neurons in age-related macular degeneration. Invest Ophthalmol Vis Sci 2001; 42: 795-803.

11 Glovinsky Y, Quigley HA, Dunkelberger GR. Retinal ganglion cell loss is size dependent in experimental glaucoma. Invest Ophthalmol Vis Sci 1991; 32: 484-491.

12 Desatnik H, Quigley HA, Glovinsky Y. Study of central retinal ganglion cell loss in experimental glaucoma in monkey eyes. J Glaucoma 1996; 5: 46-53.

13 Yucel YH, Gupta N, Kalichman MW, Mizisin AP, Hare W, de Souza Lima $\mathrm{M}$ et al. Relationship of optic disc topography to optic nerve fibre number in glaucoma. Arch Ophthalmol 1998; 116: 493-497.

14 Balazsi AG, Rootman J, Drance SM, Schulzer M, Douglas GR. The effect of age on the nerve fibre population of the human optic nerve. Am J Ophthalmol 1984; 97: 760-766.

15 Repka MX, Quigley HA. The effect of age on normal human optic nerve fibre number and diameter. Ophthalmology 1989; 96: $26-32$.
16 Mikelberg FS, Drance SM, Schulzer M, Yidegiligne HM, Weis MM. The normal human optic nerve. Axon count and axon diameter distribution. Ophthalmology 1989; 96: 1325-1328.

17 Mikelberg FS, Yidegiligne HM, Schulzer M. Optic nerve axon count and axon diameter in patients with ocular hypertension and normal visual fields. Ophthalmology 1995; 102: $342-348$

18 Radius RL, Anderson DR. The course of axons through the retina and optic nerve head. Arch Ophthalmol 1979; 97: 1154-1158.

19 Jonas JB, Muller-Bergh JA, Schlotzer-Schrehardt UM, Naumann GO. Histomorphometry of the human optic nerve. Invest Ophthalmol Vis Sci 1990; 31: 736-744.

20 Funaki S, Shirakashi M, Abe H. Relation between size of optic disc and thickness of retinal nerve fibre layer in normal subjects. Br J Ophthalmol 1998; 82: 1242-1245.

21 Jonas JB, Hayreh SS. Localised retinal nerve fibre layer defects in chronic experimental high pressure glaucoma in rhesus monkeys. Br J Ophthalmol 1999; 83: 1291-1295.

22 Ogden TE. Nerve fibre layer of the macaque retina: retinotopic organization. Invest Ophthalmol Vis Sci 1983; 24: 85-98

23 Ogden TE. Nerve fiber layer of the owl monkey retina: retinotopic organization. Invest Ophthalmol Vis Sci 1983; 24: 265-269.

24 Ogden TE. Nerve fiber layer of the primate retina: morphometric analysis. Invest Ophthalmol Vis Sci 1984; 25: 19-29.

25 Varma R, Skaf M, Barron E. Retinal nerve fiber layer thickness in normal human eyes. Ophthalmology 1996; 103: 2114-2119.

26 Ogden TE. Nerve fiber layer of the primate retina: thickness and glial content. Vision Res 1983; 23: 581-587.

27 Dichtl A, Jonas JB, Naumann GO. Retinal nerve fiber layer thickness in human eyes. Graefes Arch Clin Exp Ophthalmol 1999; 237: 474-479.

28 Quigley HA, Addicks EM. Quantitative studies of retinal nerve fiber layer defects. Arch Ophthalmol 1982; 100: 807-814.

29 Morgan JE, Waldock A, Jeffery G, Cowey A. Retinal nerve fibre layer polarimetry: histological and clinical comparison. Br J Ophthalmol 1998; 82: 684-690.

30 Abramson DH, Schefler AC, Almeida D, Folberg R. Optic nerve tissue shrinkage during pathologic processing after enucleation for retinoblastoma. Arch Ophthalmol 2003; 121: 73-75.

31 Radius RL. Thickness of the retinal nerve fiber layer in primate eyes. Arch Ophthalmol 1980; 98: 1625-1629.

32 Chauhan DS, Marshall J. The interpretation of optical coherence tomography images of the retina. Invest Ophthalmol Vis Sci 1999; 40: 2332-2342. 